\title{
Three Cases of Nivolumab Plus Ipilimumab Therapy in Haemodialysis Patients With Metastatic Renal Cell Carcinoma
}

\author{
YUKI KOBARI ${ }^{1}$, KAZUHIKO YOSHIDA ${ }^{1}$, JUNPEI IIZUKA ${ }^{1}$, TSUNENORI KONDO ${ }^{2}$, \\ HIDEKI ISHIDA ${ }^{1}$, KAZUNARI TANABE $^{1}$ and TOSHIO TAKAGI ${ }^{1}$ \\ ${ }^{1}$ Department of Urology, Tokyo Women's Medical University Hospital, Tokyo, Japan; \\ ${ }^{2}$ Department of Urology, Tokyo Women's Medical University Medical Center East, Tokyo, Japan
}

\begin{abstract}
Background: Although the CheckMate 214 trial affirmed the effectiveness of nivolumab-ipilimumab combination therapy in advanced or metastatic renal cell carcinoma $(m R C C)$, its safety and efficacy in patients with end-stage renal disease (ESRD) on haemodialysis remains unexplored. Case Report: All patients were male and underwent $m R C C$ treatment with partial nephrectomy or nephrectomy. They had ESRD and were undergoing haemodialysis. Cases 1 and 2 showed lymph node and lung metastases after initial surgery and received nivolumabipilimumab therapy. Case 1 had grade 3 adrenal insufficiency after four courses, which was controlled with steroids. Case 2 did not experience adverse events. Both were well controlled with complete $(C R)$ or partial response $(P R)$. Case 3 suffered local recurrence after nephrectomy and received combination therapy. Grade 3 adrenal insufficiency occurred following three courses, and tumour size did not change remarkably. Conclusion: Nivolumabipilimumab combination therapy can effectively treat $m R C C$ patients with ESRD undergoing haemodialysis.
\end{abstract}

The treatment of metastatic renal cell carcinoma (mRCC) changed significantly with the arrival of immune checkpoint inhibitors (ICIs). For patients classified as having intermediate- and poor-risk according to International Metastatic RCC Database Consortium (IMDC) (1), nivolumab-ipilimumab combination therapy offers significantly higher overall survival (OS) rates than sunitinib

This article is freely accessible online.

Correspondence to: Kazuhiko Yoshida, MD, PhD, Department of Urology, Tokyo Women's Medical University Hospital, Tokyo, Japan. Tel: +81 333538111, e-mail: kzyoshida1@yahoo.co.jp

Key Words: metastatic renal cell carcinoma, haemodialysis, nivolumab, ipilimumab. as shown in the CheckMate 214 trial (2). However, this clinical trial excluded patients with ESRD undergoing haemodialysis. The incidence of RCC in haemodialysis patients is higher than in the general population (3). In addition, patients with mRCC are more commonly encountered in real-world clinical practice. However, there is a lack of established treatment strategies for mRCC patients undergoing haemodialysis.

Herein, we report findings from three patients with mRCC undergoing haemodialysis treated with nivolumabipilimumab combination therapy.

\section{Case Report}

The characteristics and treatment outcomes of the three patients are summarized in Table I.

Case 1. A 70-year-old-male was diagnosed with a right renal tumour during a screening abdominal ultrasound examination and was referred to our hospital for further assessment. He had stage 4 chronic kidney disease (CKD) due to renal sclerosis. Enhanced computed tomography (CT) revealed suspicious RCC with a diameter of $55 \mathrm{~mm}$ located in the upper pole of the right kidney. He had undergone robot-assisted partial nephrectomy in August 2018. Pathological findings revealed clear cell RCC, pT1b, and a negative surgical margin. Follow-up CT in September 2019 showed metastasis on back skin and, subsequently, metastasectomy was performed. The pathological diagnosis was metastatic RCC. Paraaortic lymph node (LN) metastasis occurred in December 2019, and lung metastasis was observed in February 2020. His renal function worsened, and haemodialysis was initiated in April 2020. Because of enlarging LN and lung metastases, he was treated with nivolumab (240 $\mathrm{mg})$ plus ipilimumab $(1 \mathrm{mg} / \mathrm{kg})$ therapy every 3 weeks for IMDC intermediate risk $\mathrm{mRCC}$ in May 2020. After 4 courses of nivolumab-ipilimumab therapy, LN (Figure 1A) and lung lesions showed CR. 
Table I. Case summary,

\begin{tabular}{lccc}
\hline & Case 1 & Case 2 & Case 3 \\
\hline Gender & Male & Male & Male \\
Age (years) & 70 & 63 & 40 \\
BMI $\left(\mathrm{kg} / \mathrm{m}^{2}\right.$ ) & 24.9 & 20.8 & 20.5 \\
HD duration & $1 \mathrm{~m}$ & $25 \mathrm{y} 4 \mathrm{~m}$ & $2 \mathrm{y} 11 \mathrm{~m}$ \\
Tumour side & Right & Bilateral & Left \\
Clinical stage & cT1bN0M0 & cT1aN0M0 & cT1aN0M0 \\
Operation & Partial nephrectomy & Nephrectomy & Nephrectomy \\
Pathology & Clear cell pT1b G2>G3 ly0 & L: papillary pT1a G2 ly0 & Clear cell pT1a G3 ly0 \\
IMDC classification & R: Clear cell pT1a G2 ly0 & Intermediate risk \\
Metastasis site & Intermediate risk & Intermediate risk & Local recurrence \\
Time from diagnosis to combination therapy & Skin, LN, Lung & Lung, LN & 2y 11 m \\
Target lesions status after combination therapy & 1y 8 m & CR & SD \\
irAE & Adrenal insufficiency (grade 3) & none & Adrenal insufficiency (grade 3) \\
Current treatment & Observation (durable response) & Nivolumab monotherapy & Nivolumab monotherapy \\
\hline
\end{tabular}

BMI: Body mass index; HD: haemodialysis; IMDC: International Metastatic RCC Database Consortium; LN: lymph node; irAE: immune-related adverse events.

Subsequently, according to the Common Terminology Criteria for Adverse Events (CTCAE) version 5.0 a grade 3 central adrenal insufficiency, which is an immunerelated adverse event (irAE), occurred. Adrenal insufficiency was controlled using oral steroids. He was treated with nivolumab (480 mg) every 4 weeks. He repeatedly suffered adrenal insufficiency due to a urinary tract infection. Therefore, nivolumab therapy was discontinued. However, the disease remains under control, and his CR status has remained for seven months after stopping nivolumab.

Case 2. A 63-year-old man was referred to our hospital for bilateral kidney tumours diagnosed using regular enhanced CT. He had been undergoing haemodialysis for ESRD due to chronic glomerulonephritis since 1994. Left laparoscopic nephrectomy was performed in April 2011, and a right nephrectomy was performed in June 2011. Pathological findings showed papillary RCC (pT1a) on the left and clear cell RCC (pT1a) on the right; both surgical margins were negative. Following surgery, the patient stopped hospital visits. In September 2020, he visited our hospital with lung and LN metastases diagnosed on CT at a maintenance dialysis hospital. He was treated with nivolumab $(240 \mathrm{mg}$ ) and ipilimumab (1 $\mathrm{mg} / \mathrm{kg})$ every 3 weeks for IMDC intermediate risk mRCC. The lung and LN metastases decreased in size (PR) without any adverse events (Figure 1B). Following 4 courses of combination therapy, nivolumab therapy was started every 4 weeks. So far, he has received five courses of nivolumab. His tumour growth remains controlled with PR status, and no adverse events have been noted until writing this report.
Case 3. A 40-year-old man had been undergoing haemodialysis for end-stage renal disease (ESRD), whose aetiology was unknown, since 2008. He had undergone laparoscopic nephrectomy for a left renal cystic tumour at another hospital in 2018, and the renal cyst was ruptured during the surgery. The pathological diagnosis was clear cell RCC (pT1a), and the surgical margin was negative. A followup CT in December 2019 revealed soft tissue masses in the left retroperitoneal space. These tumours enlarged by January 2021 , and he was referred to our hospital for treatment. He was started on nivolumab $(240 \mathrm{mg})$ and ipilimumab $(1 \mathrm{mg} / \mathrm{kg})$ every 3 weeks for IMDC intermediate risk mRCC. Following three courses of combination therapy, he was admitted to the emergency department due to high fever and fatigue. Grade 3 central adrenal insufficiency was diagnosed during a further examination, and the patient received steroid therapy. The size of the locally recurrent tumours did not change significantly, suggesting a stable disease (SD). After controlling the adrenal insufficiency using steroids, we initiated nivolumab monotherapy (480 mg/4 weeks). He was treated with three courses of nivolumab and has attained SD status without additional AEs.

\section{Discussion}

The CheckMate 214 trial in 2018 demonstrated the effectiveness of first-line treatment with nivolumabipilimumab combination therapy for advanced or metastatic clear cell RCC. Combination therapy can also be helpful for non-clear cell RCC (4), even with cardiac metastases (5). In contrast, combination therapy for papillary RCC had a high rate of progressive disease (PD) and poorer progression-free 

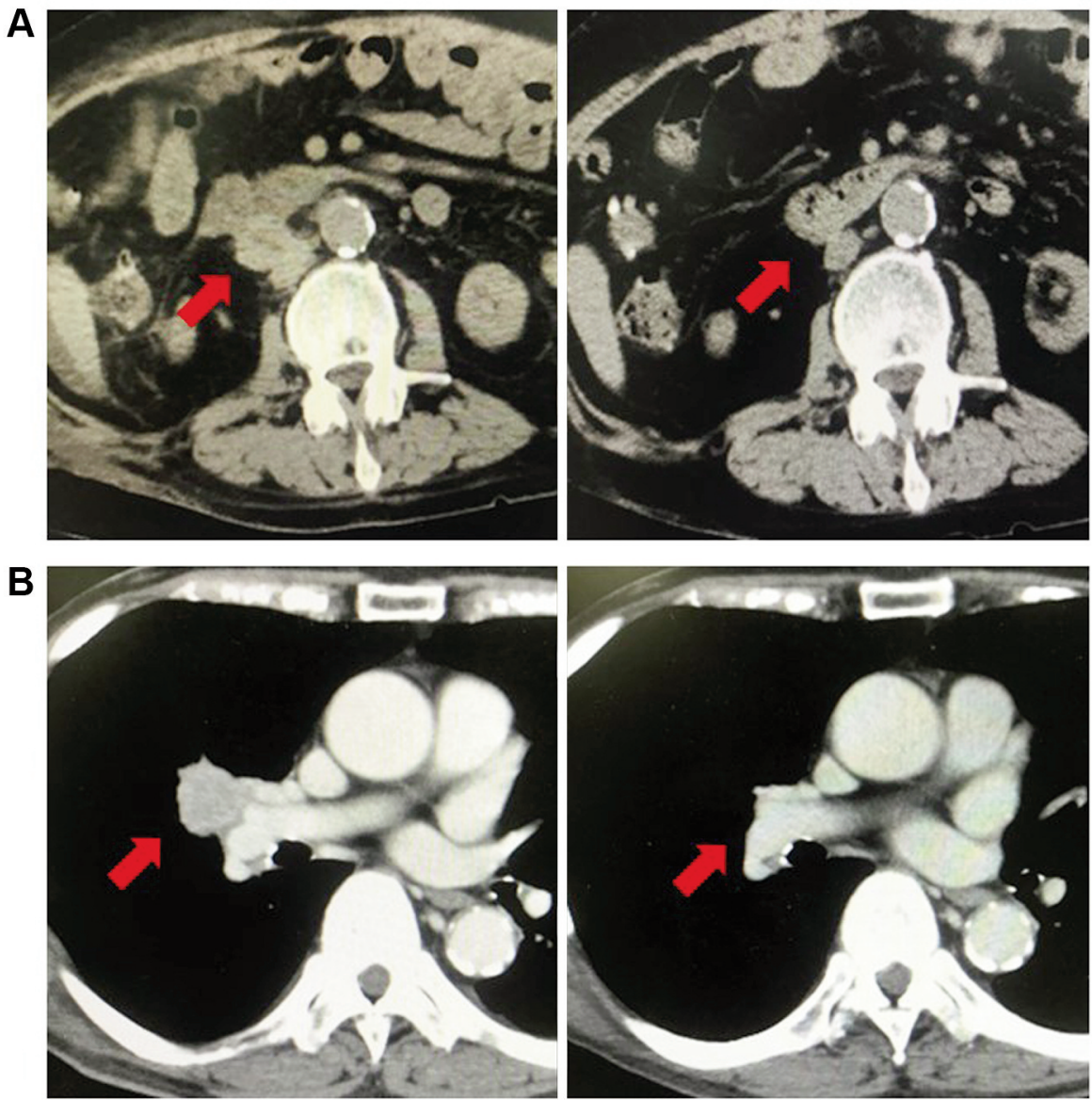

Figure 1. Computed tomography imaging findings for cases 1 and 2. (A) The image shows lymph node metastases before treatment and after 4 nivolumab plus ipilimumab therapy courses. Lymph node metastases were not observed. (B) The image shows lung hilar lymph node metastases before treatment and after four courses of combination therapy. Lymph node metastases were not observed.

survival than that of clear cell RCC (6). However, both studies excluded haemodialysis patients. Therefore, there is no established treatment strategy for mRCC patients with ESRD undergoing haemodialysis.

There are several case reports of ESRD patients with mRCC treated using ICIs. A 68-year-old man with mRCC receiving haemodialysis for 20 years reportedly had a controlled disease with long-term use of nivolumab without immune-related adverse events (irAEs) after sunitinib was considered ineffective (7). In another case, a 77-year-old man with mRCC undergoing haemodialysis, nivolumab was used as the fourth-line treatment. He suffered respiratory failure two weeks after initiating nivolumab and required intubation. His case was considered as tumour pseudoprogression, and his condition improved with antibiotics and diuresis, allowing extubation after 2 days. Eight months after initiating nivolumab, the patient's cancer attained PR status (8). Cavalcante et al. reported two cases of haemodialysis patients with metastatic melanoma treated using ipilimumab (9). Although grade 1 itching appeared, ipilimumab therapy helped achieve PR status in the first case, and the other case achieved CR status with grade 3 bullous pemphigoid. It was said that irAEs were associated with the prognosis of patients with mRCC treated ICIs. Moreover, patients with irAEs had significantly longer OS and progression-free survival than those without (10).

In our case, the tumour was well controlled in every patient with either CR, PR, or SD status. Because our cases were clear cell or papillary RCC, the effectiveness of combination therapy for the patients with acquired cystic disease associated RCC, which occurred in heamodialysis patients as well, was unclear. Moreover, we have to consider site-specific responses to nivolumab may vary depending on the organ that has metastasized (11).

However, in terms of adverse events, two of the three patients suffered grade 3 central adrenal insufficiency, which 
was controllable using steroid therapy. The adrenal insufficiency recurred in the first patient due to infections.

A systematic review of the literature found that patients receiving combination ICIs had a higher incidence of adrenal insufficiency than those receiving ICI monotherapy (12-14). However, this review did not discuss cases of haemodialysis patients. Several patients with central adrenal insufficiency induced by ipilimumab reportedly need to continue steroid therapy due to persistent central adrenal insufficiency (15). Patients with ESRD can have a higher incidence of infections than the general population because of acquired immune deficiency (16), and steroids can further increase the risk of infections (17). Considering these observations, haemodialysis patients have a considerable risk of infections when on steroids. Based on our case findings, infections were thought to have induced relative adrenal insufficiency, as in our first case. In patients undergoing heamodialysis and receiving combination ICIs, infections or any stress might induce relative adrenal insufficiency. However, comparing $\mathrm{AE}$ in haemodialysis patients remains challenging due to the limited number of case reports.

Haemodialysis patients receiving steroids due to irAEs must be carefully followed up. The nivolumab-ipilimumab combination therapy for mRCC patients with ESRD can help manage the disease effectively.

\section{Conclusion}

We reported the cases of three patients with mRCC undergoing haemodialysis treated using nivolumab-ipilimumab combination therapy. We consider that early detection of irAEs and proper treatment is critical, especially in haemodialysis patients treated with combination therapy. The nivolumabipilimumab combination therapy can be considered effective even in haemodialysis patients with mRCC.

\section{Conflicts of Interest}

All Authors have no conflicts of interest to declare in relation to this study.

\section{Authors' Contributions}

YK wrote this manuscript and provided a table and figure. YK, KY, JI, TK, HI, KT and TT cared for the patients and administered the combination therapy. All Authors read and approved the final manuscript.

\section{References}

1 Heng DY, Xie W, Regan MM, Harshman LC, Bjarnason GA, Vaishampayan UN, Mackenzie M, Wood L, Donskov F, Tan MH, Rha SY, Agarwal N, Kollmannsberger C, Rini BI and Choueiri TK: External validation and comparison with other models of the International Metastatic Renal-Cell Carcinoma Database Consortium prognostic model: a population-based study. Lancet Oncol 14(2): 141-148, 2013. PMID: 23312463. DOI: 10.1016/S1470-2045(12)70559-4

2 Motzer RJ, Tannir NM, McDermott DF, Arén Frontera O, Melichar B, Choueiri TK, Plimack ER, Barthélémy P, Porta C, George S, Powles T, Donskov F, Neiman V, Kollmannsberger CK, Salman P, Gurney H, Hawkins R, Ravaud A, Grimm MO, Bracarda S, Barrios CH, Tomita Y, Castellano D, Rini BI, Chen AC, Mekan S, McHenry MB, Wind-Rotolo M, Doan J, Sharma P, Hammers HJ, Escudier B and CheckMate 214 Investigators: Nivolumab plus ipilimumab versus sunitinib in advanced renalcell carcinoma. N Engl J Med 378(14): 1277-1290, 2018. PMID: 29562145. DOI: $10.1056 /$ NEJMoa1712126

3 Kondo T, Sasa N, Yamada H, Takagi T, Iizuka J, Kobayashi H, Yoshida K, Fukuda H, Ishihara H, Tanabe $\mathrm{K}$ and Tsuzuki T: Acquired cystic disease-associated renal cell carcinoma is the most common subtype in long-term dialyzed patients: Central pathology results according to the 2016 WHO classification in a multi-institutional study. Pathol Int 68(10): 543-549, 2018. PMID: 30187581. DOI: 10.1111/pin.12718

4 Gupta R, Ornstein MC, Li H, Allman KD, Wood LS, Gilligan T, Garcia JA, Merveldt DV, Hammers HJ and Rini BI: Clinical activity of ipilimumab plus nivolumab in patients with metastatic non-clear cell renal cell carcinoma. Clin Genitourin Cancer 18(6): 429-435, 2020. PMID: 32800717. DOI: 10.1016/j.clgc.2019.11.012

5 Shirotake S, Umezawa Y, Okabe T, Kaneko GO, Kanao K, Nishimoto $\mathrm{K}$ and Oyama M: Efficacy of nivolumab plus ipilimumab in a patient with renal cell carcinoma concomitant with cardiac metastasis: a case report. In Vivo 34(3): 1475-1480, 2020. PMID: 32354949. DOI: 10.21873/invivo.11932

6 Tachibana H, Kondo T, Ishihara H, Fukuda H, Yoshida K, Takagi T, Izuka J, Kobayashi H and Tanabe K: Modest efficacy of nivolumab plus ipilimumab in patients with papillary renal cell carcinoma. Jpn J Clin Oncol 51(4): 646-653, 2021. PMID: 33212488. DOI: 10.1093/jjco/hyaa229

7 Morinaga R, Kawahara T, Miyoshi Y, Yao M and Uemura H: Longer control of nivolumab in metastatic renal cell carcinoma patients with end-stage kidney disease on dialysis. Case Rep Oncol 12(2): 608-612, 2019. PMID: 31543776. DOI: 10.1159/000501768

8 Carlo MI and Feldman DR: Response to nivolumab in a patient with metastatic clear cell renal cell carcinoma and end-stage renal disease on dialysis. Eur Urol 70(6): 1082-1083, 2016. PMID: 27311362. DOI: 10.1016/j.eururo.2016.05.040

9 Cavalcante L, Amin A and Lutzky J: Ipilimumab was safe and effective in two patients with metastatic melanoma and endstage renal disease. Cancer Manag Res 7: 47-50, 2015. PMID: 25632239. DOI: $10.2147 / C M A R . S 73389$

10 Fiala O, Sorejs O, Sustr J, Kucera R, Topolcan O and Finek J: Immune-related adverse effects and outcome of patients with cancer treated with immune checkpoint inhibitors. Anticancer Res 40(3): 1219-1227, 2020. PMID: 32132018. DOI: 10.21873/ anticanres. 14063

11 Negishi T, Furubayashi N, Nakagawa T, Nishiyama N, Kitamura H, Hori Y, Kuroiwa K, Son Y, Seki N, Tomoda T, Okajima E and Nakamura M: Site-specific response to nivolumab in renal cell carcinoma. Anticancer Res 41(3): 1539-1545, 2021. PMID: 33788747. DOI: 10.21873 /anticanres.14913

12 Brahmer JR, Lacchetti C and Thompson JA: Management of immune-related adverse events in patients treated with immune 
checkpoint inhibitor therapy: American Society of Clinical Oncology clinical practice guideline summary. J Oncol Pract 14(4): 247-249, 2018. PMID: 29517954. DOI: 10.1200/JOP.18. 00005

13 Salinas C, Renner A, Rojas C, Samtani S and Burotto M: Primary adrenal insufficiency during immune checkpoint inhibitor treatment: Case reports and review of the literature. Case Rep Oncol 13(2): 621-626, 2020. PMID: 32595473. DOI: $10.1159 / 000507652$

14 Corsello SM, Barnabei A, Marchetti P, De Vecchis L, Salvatori $\mathrm{R}$ and Torino F: Endocrine side effects induced by immune checkpoint inhibitors. J Clin Endocrinol Metab 98(4): 13611375, 2013. PMID: 23471977. DOI: 10.1210/jc.2012-4075

15 Mahzari M, Liu D, Arnaout A and Lochnan H: Immune checkpoint inhibitor therapy associated hypophysitis. Clin Med Insights Endocrinol Diabetes 8: 21-28, 2015. PMID: 25861234. DOI: $10.4137 / C M E D . S 22469$
16 Sarnak MJ and Jaber BL: Mortality caused by sepsis in patients with end-stage renal disease compared with the general population. Kidney Int 58(4): 1758-1764, 2000. PMID: 11012910. DOI: $10.1111 /$ j.1523-1755.2000.00337.x

17 Youssef J, Novosad SA and Winthrop KL: Infection risk and safety of corticosteroid use. Rheum Dis Clin North Am 42(1): 157-76, ix-x, 2016. PMID: 26611557. DOI: 10.1016/j.rdc.2015. 08.004

Received July 30, 2021

Revised August 27, 2021

Accepted September 2, 2021 